\title{
BUILDING FOOTPRINT EXTRACTION FROM HIGH RESOLUTION SATELLITE IMAGERY USING SEGMENTATION
}

\author{
Shweta Khatriker ${ }^{1, *}$, Minakshi Kumar ${ }^{1}$ \\ Indian Institute of Remote Sensing, Dehradun, India - shwetakhatriker29@gmail.com, minakshi@iirs.gov.in
}

Commission V, WG V/7

KEY WORDS: Segmentation, Building Extraction, Multi-Resolution Algorithm, Rule-Based Feature Extraction, High Resolution Satellite Imagery

\begin{abstract}
:
Identification and mapping of urban features such as buildings and roads are an important task for cartographers and urban planners. High resolution satellite imagery supports the efficient extraction of manmade objects. For the planning and designing of Smart cities, building footprint information is an essential component, and geospatial technologies helps in creating this large mass of data inputs for designing and planning of smart cities. In this study segmentation approach is followed for building extraction. For extraction of buildings especially from the high resolution imagery, number of various semiautomatic and automatic methods have been developed till date to reduce the time and efforts required in manual building mapping. In this study, two semiautomatic image segmentation techniques are used for building extraction from high resolution imagery using algorithms- Multi-resolution segmentation and Rule based feature extraction, which are applied on Worldview 2 (2010) imagery of Dehradun area. The segmented image were further classified to extract buildings from the segmented image features. The study identify the usefulness of both the methods in building extraction and finds the optimum set of rules for extracting buildings from high resolution data sets. The True Positive Rate using Rule based feature extraction is $88.11 \%$ compared to $85.46 \%$ from Multi-resolution segmentation algorithm. The False Negative Rate (FNR) of Multi-resolution segmentation algorithm (16.5\%.) is very less compared to Rule based feature extraction (67.5\%). In the study the buildings were extracted with the accuracy of $88.9 \%$.
\end{abstract}

\section{INTRODUCTION}

\subsection{Background}

Building footprint is the basic data required prior to any development or planning work. High resolution data support the efficient extraction of manmade objects and helps in planning and mapping activities. Manual extraction and digitization of urban features from imagery are time consuming and tedious process. Segmentation method is comparatively inexpensive and time saving for extraction of urban features. For extraction of buildings, especially from the high-resolution imagery, number of various semiautomatic and automatic methods have been developed till date to reduce the time and efforts required in manual building mapping. But this is a challenging task due to complex shapes of building and the similarity in shape and sizes of other urban features like roads etc. (Singh et al., 2012). Building extraction and identification is one of the important issues for any urban development work. The importance of buildings for planning, economic, environmental and demographic studies, generate the need to derive an effective and efficient methodology for accurate extraction of buildings. It is necessary to identify an accurate and reliable method or parameters for extraction of buildings from high resolution satellite imagery as alternate methods such as manual digitization or automatic extraction methods are cost and time intensive. This study answers the questions such as, What are the two multi resolution and rule based features extraction algorithms? What are the accuracy of the features extraction using both techniques?

\section{BUILDING EXTRACTION}

\subsection{Segmentation approach}

Segmentation is defined as delineation of entire digital image into number of segments or set of pixels, the goal is to enhance the present objects of the image into something more meaningful and required. The image segmentation methods generally are used for recognizing and differentiating various features of the image. Image segmentation process divide pixels of similar properties such as spectral reflectance, texture or area etc. into one group or region (Jiang et al., 2008). For any development activities, city planning, transportation planning and disaster management strategies etc., buildings are the basic element for analysis. All these tasks require accurate and timely mapping of urban features especially buildings.

With the advancement of technologies the process of buildings extraction has become less manual and more automatic, but automatic extraction methods are more expensive and time consuming compared to semi-automatic methods. Building extraction depends on various factors as described by Singh et al. (2012) "Building extraction also depend on the quality of image, quality of brightness, weather conditions in which image has taken, that's why it is not possible to obtain $100 \%$ results (Singh et al., 2012). So by minimizing the dependency of building extraction methods on these factors we can achieve better results" (Singh et al., 2012). Semi-automatic methods such as Multiresolution from segmentation tool of eCognition and rule based features extraction module of ENVI are some examples of such methods of building extraction. The other challenges faced

${ }^{*}$ Corresponding author 
during process of extraction of features are the suitable combination of rule sets. These rule sets are the unique properties exhibited by different features which differentiate them from each other. Formation of suitable rule sets for extraction of buildings are very important as many other features have similar spectral properties similar to buildings which affects the accuracy of features extraction. Similarly accuracy assessment is other important task of feature extraction.

\section{SEGMENTATION}

\subsection{Method of feature extraction}

Features extraction can also be called as finding object in an image. This process allows us to isolate the only objects we are interested in, for example buildings, roads etc. The feature extraction from an urban areas are dependent on number of properties of the objects such as material, texture, density etc. (Belgiu and Drăguţ, 2014). These parameters plays an important role in final output of extraction. The spatial and spectral properties are the two important factors for extraction. Spatial deals with space, how combination of neighborhood pixels are defined by location of that pixels. Scale is also an important parameter and dependent on the resolution of the data, meaning high resolution data higher accuracies of extraction. Similarly methods of extraction of features also varies with respect to resolution of the sensors. Pixels based extraction method is used for medium and low resolution dataset. For extraction of features from high resolution datasets, object based image analysis is used. Spectral Properties deals with the unique spectral signatures of features and behavior of objects in different ranges of band values in a multispectral imagery. The similarity in spectral properties of some urban features result in misclassification of urban land cover classes for example buildings and dry riverbed both resemble similar because of the similar spectral response.

The feature extraction is done using three approaches - Manual or Non-Automatic, Semi-Automatic and Automatic methods. Methods such as object based semantic approach, Certainty voting etc. are Non Automatic methods of extraction. Automatic methods of feature extraction involves various morphological operators. Jumlesha et al., (2012) have used mathematical operators to extract urban features such as roads and buildings from satellite imagery in Matlab environment. Benediktsson, Pesaresi and Arnason, (2003) have proposed an approach for urban area classification using morphological operators and neural network where morphological operators are used for extraction of features and neural network approach are used to classify the extracted features. Semi-Automatic method involves segmentation of image based on factors specified by the user and classification of the segmented image to extract the desired features.

\section{STUDY AREA}

\subsection{Dehradun}

The study area is located in the Dehradun city. Dehradun is the capital of Uttarakhand state of India shown in Fig.1. Located around $236 \mathrm{~km}$ north of the capital of India, Delhi, Dehradun is being developed as one of the counter magnet of the National Capital Region to decrease the population and infrastructure pressure on Delhi. It is also proposed to be developed as smart city under smart city mission of government of India. It has environmental and religious significance as number of famous religious places are present here as well as famous rivers and vegetation species are found in the forests of Dehradun (Uttarakhand, 2018). The average elevation of the city is $450 \mathrm{~m}$ above mean sea level. The climate type of Dehradun is humid subtropical. According to the Census of India, 2011, the population of Dehradun city is 5, 69,578. It is a central part of tourism stoppage as number of famous tourist spots are located near it. Flow of large mass of population is very normal for the city. Analysis of urban infrastructure availability and to make sure accessibility is an important part of the management work of city administrators and planners.

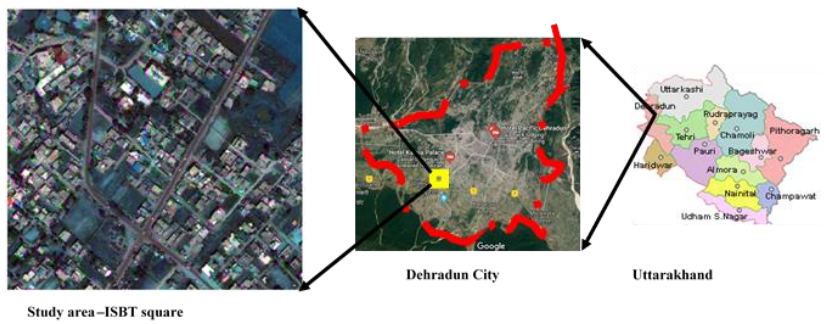

Figure 1 Study area, Dehradun

\subsection{Data sets}

Satellite imagery from Worldview 2 of year 2010 is used as input data for the analysis and building extraction. Panchromatic image of Worldview has spatial resolution of 0.5 meters and multispectral image of Worldview 2 has spatial resolution of 2 meters. To get high spatial and spectral resolutions data set, fusion of both the images is performed. The resultant multispectral image has 0.5 meters of spatial resolution (Figure 2). For the extraction of buildings two software are used, eCognition Developer 9.1 and ENVI 5.0.
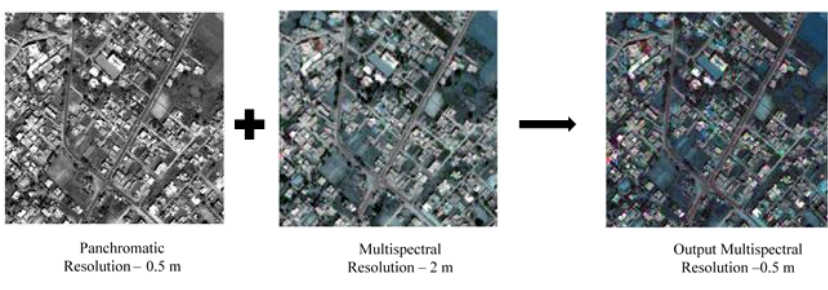

Figure 2 Worldview Data sets used for study

\subsection{Methodology}

The first step of the process involved getting a high resolution data set. To get a high resolution multispectral image, panchromatic and multispectral satellite images of Worldview 2 are fused using Principal Component Analysis method and bilinear interpolation method of resampling is used. PCA method was used as the available worldview data sets, had only four bands, and PCA method uses all the four bands on the other hand all the other methods uses only three bands. The second stage of the analysis is consist of Segmentation of image to get regions of different image objects. Two segmentation modules/software have been used, eCognition 9.1 and ENVI 5.0. eCognition Developer's Multiresolution algorithms and Rule based feature extraction algorithm of ENVI were used for segmentation of image. Different sets of rules were adopted for the segmentation and classification of buildings in both the software. The process of selection of rule sets involved literature review on the method and hit and trial method along with visual interpretation. Segmented image were classified using classification algorithm 
in eCognition and spectral mean and compactness parameters in ENVI.

The next step of the analysis involved accuracy assessment of extracted buildings in both the software. For the accuracy assessment two approaches were adopted. Confusion matrix for all the classes of land covers were generated in eCognition. Total 20 samples of buildings along with 15 samples of vegetation and 5 samples from road were taken to perform accuracy assessment in eCognition. To assess and compare the results another approach proposed by (Jamet, 1997) is used. True Positive Rate, False Positive Rate and False Negative Rate were calculated for the extracted buildings. True Positive Rate is defined as the number of correctly classified pixels to that of reference at correct place. False Positive Rate is the number of incorrectly classified pixels to that of reference, False Negative Rate is defined as the number of correctly identified pixels but outside the reference object or at wrong place. The methodology process is shown in Fig.3.

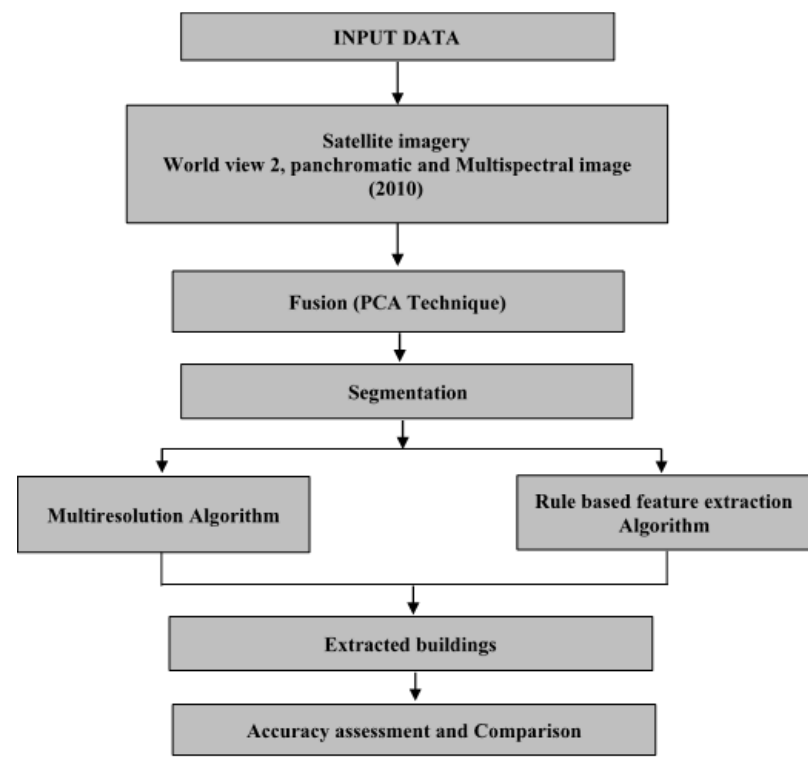

Figure 3 Methodology chart

The output data from ENVI and eCognition were exported into shapefiles and with the help of ARCMAP 10.1 software where the area calculation and accuracy assessment were performed. The comparison of both the eCognition Developer's Multiresolution algorithms and Rule based feature extraction algorithm of ENVI are made based on these TPR, FPR and FNR and percentage accuracies are calculated.

\section{RESULT AND DISCUSSION}

\subsection{Feature extraction using Multi-Resolution Algorithm}

Multi-resolution segmentation algorithm of eCognition follows a bottom up approach of region merging technique. The method allows users to apply optimization procedure which minimizes the average heterogeneity of image objects for a given resolution. Parameters such as scale, shape and compactness influences the final segmentation output.

5.1.1 Rules and algorithms: The various set of rules during segmentation and classification were used. The Segmentation algorithm of Multiresolution Segmentation is used, with scale of segmentation equal to 60 out of 100 . Homogeneity criterion with value of 0.7 as shape and 0.5 as compactness are used (Figure 4 ). The segmented image were further classified to extract buildings out of other land cover features such as roads and vegetation. 1010 samples from all the classes were taken. Samples are shown in the Fig.5. These three classes were classified using classification algorithm and the classification rules of Object features such as Layer value, Mean Brightness; Geometry, Shape Density ; Texture, ,Texture after Haralick (GLCM homogeneity 45- ) were used. The rule set of classification is taken from the literature review. Final classified image is shown in the Fig.6.

5.1.2 Accuracy assessment: For accuracy assessment, 20 samples of buildings and 15 of vegetation and 5 of roads were taken separately and confusion matrix was generated. Overall accuracy for the classification is $88.9 \%$. Also the producer's accuracy of extracted buildings are $85.7 \%$ which is near to satisfactory.

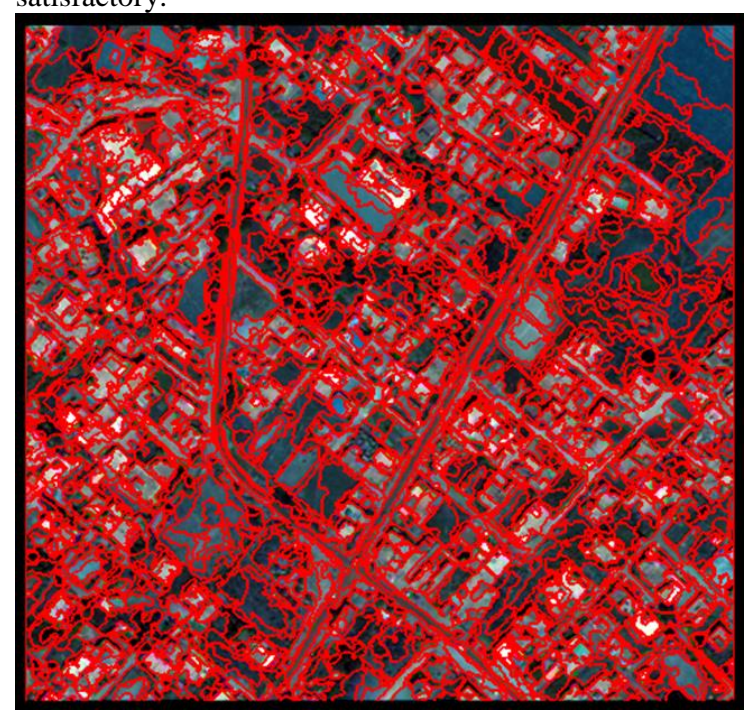

Figure 4 Segmented Image using Multi-resolution algorithm

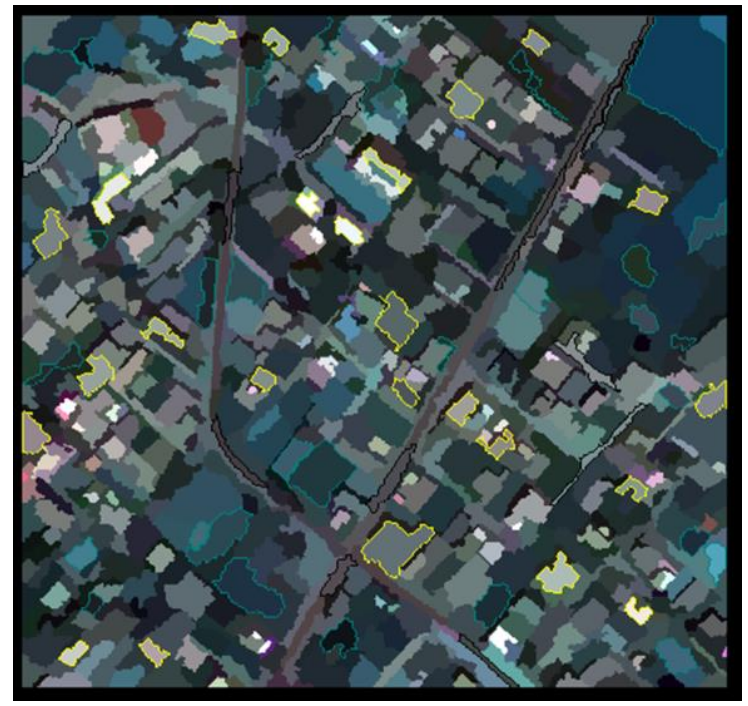

Figure 5 Samples for Classification

\subsection{Feature extraction using Rule based Feature extraction (RBFE) Algorithm}

Features extraction using Rule based approach in ENVI is based on the pixel DN values. It provides the option of specifying the ranges of $\mathrm{DN}$ values or single $\mathrm{DN}$ values for segmenting the 
image (Itt, 2008). The method allows users to specify the scale level for segmentation algorithm and merge level for merge setting algorithms for the object creation. Classification of the segmented object are performed by Rule based approach, where different sets of rules and their threshold values can be specified for extraction of desired features. This also provide a visualization option for the rules. In the study Rule based feature extraction algorithm were used for Segmentation. The method was Edge detection at scale of 25 (scale were taken after hit and trial). Full lambda schedule scale of 98 out of 100 were used as the merge setting. The classification rules to separate buildings with other land covers or features are Compactness at level more than 0.17804 (Figure 7) and Spectral Mean at level more than 323.80 (Figure 8). The extracted buildings are shown in Figure 9 $\& 10$.

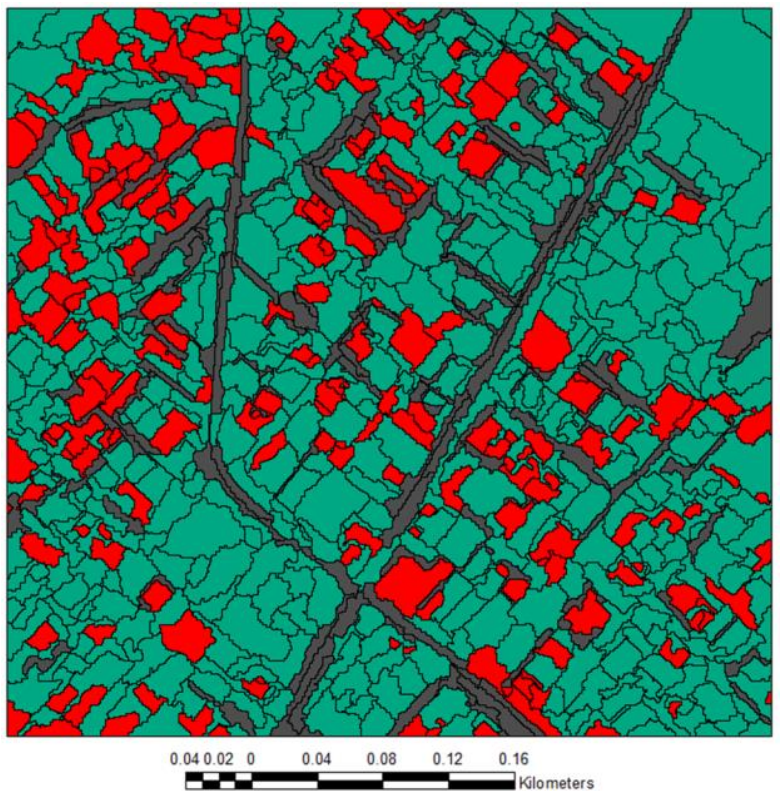

Legend

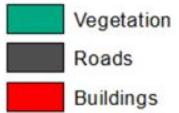

Figure 6 Classified Image using Multi-resolution algorithm

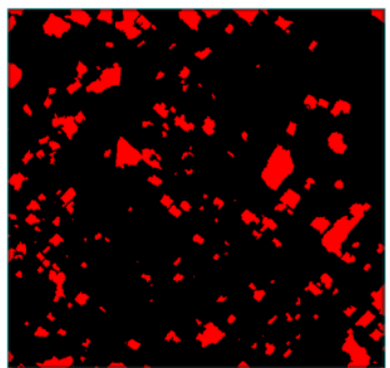

Figure 7 Building features extraction rule, Compactness

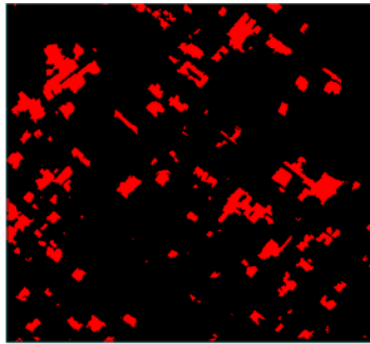

Figure 8 Building features extraction rule, Spectral Mean

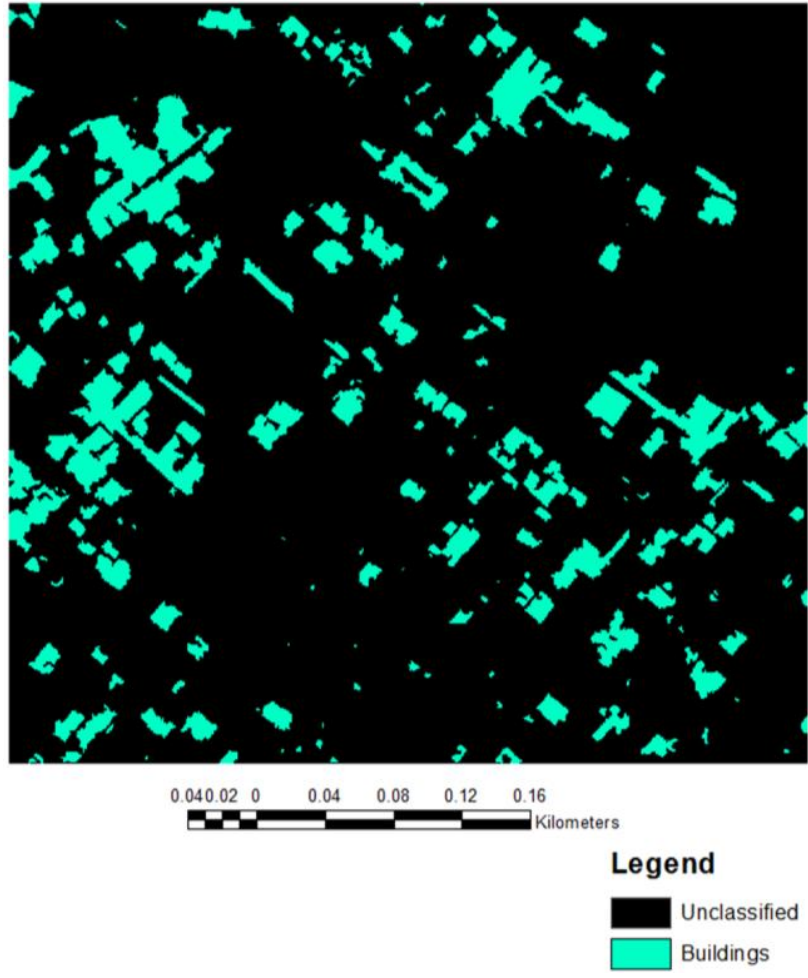

Figure 9 Extracted buildings using Rule based feature extraction algorithm

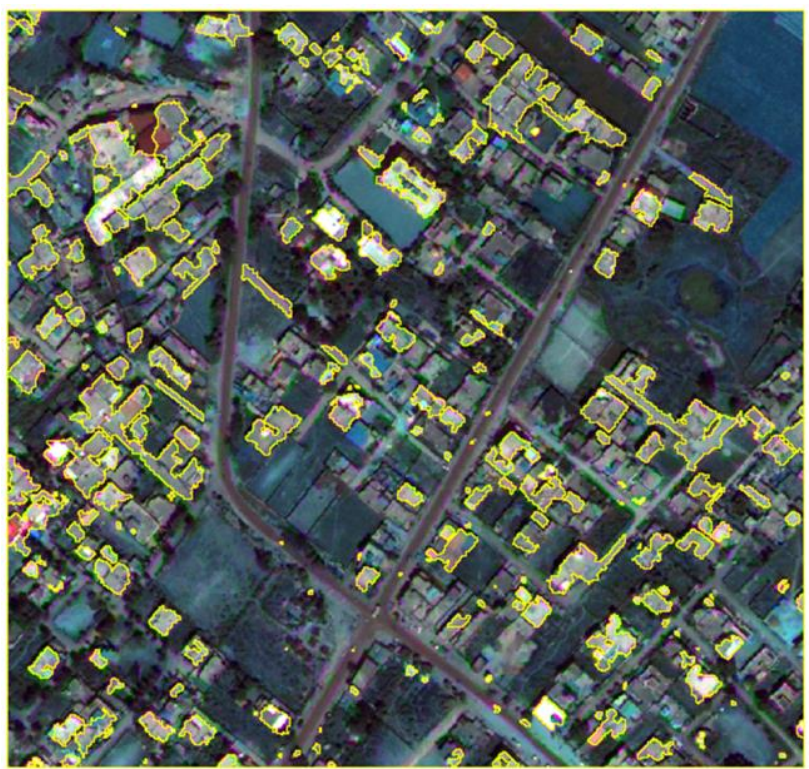

Figure 10 Extracted building footprints from RBFE on reference image

\section{ACCURACY ASSESSMENT}

For the comparison of the two segmentation techniques applied for building extraction True Positive Rate (TPR), False Positive Rate (FPR) and False Negative Rate (FNR) were calculated (Table $11 \& 12$ ) for the exported building features from both the algorithms - Multi-Resolution and Rule based feature extraction. The TPR for Rule based feature extraction is $88.11 \%$ compared 
to $85.46 \%$ using Multi-Resolution. The correctly classified buildings by Rule based algorithm are more than that by MultiResolution algorithms. The False Positive Rate (FPR) of extraction by Rule based algorithm is less than that of MultiResolution algorithms, meaning that pixels which has been incorrectly classified as buildings are less by $2.65 \%$ from MultiResolution algorithms. The False Negative Rate (FNR) of MultiResolution algorithms is very less compared to Rule based algorithm. From this we can infer that large numbers of pixels belonging to other classes than buildings has been classified as buildings by Rule based algorithm. The FNR of Rule based algorithm is $67.5 \%$ compared to that of Multi-Resolution algorithms which is very less $16.5 \%$ (Figure $13 \& 14$ ).

\begin{tabular}{|l|r|r|}
\hline & \multicolumn{2}{|c|}{ Area Sq. m. / (\%) } \\
\hline & $\begin{array}{c}\text { Rule based feature } \\
\text { extraction algorithm }\end{array}$ & $\begin{array}{c}\text { Multiresolution } \\
\text { algorithms }\end{array}$ \\
\hline $\begin{array}{l}\text { Correctly classified } \\
\text { building area }\end{array}$ & $2446.77(88.11 \%)$ & $\mathbf{2 2 9 7 . 8 3 ( 8 5 . 4 6 \% )}$ \\
\hline $\begin{array}{l}\text { Reference Building } \\
\text { area }\end{array}$ & $\mathbf{2 7 7 6 . 9 4}$ & \\
\hline $\begin{array}{l}\text { Classified Building } \\
\text { area }\end{array}$ & $\mathbf{4 3 2 1 . 2 5}$ & \\
\hline
\end{tabular}

Table 11 Comparison of results obtained from the algorithms

\begin{tabular}{|c|r|r|}
\hline Accuracy & $\begin{array}{c}\text { Multiresolution } \\
\text { algorithms (\%) }\end{array}$ & $\begin{array}{c}\text { Rule based feature } \\
\text { extraction } \\
\text { algorithm (\%) }\end{array}$ \\
\hline True Positive Rate & $\mathbf{8 8 . 1 1}$ & $\mathbf{8 5 . 4 6}$ \\
\hline False Positive Rate & 11.89 & 14.54 \\
\hline False Negative Rate & $\mathbf{6 7 . 5 0}$ & \\
\hline
\end{tabular}

Table 12 Accuracy assessment of both the algorithms

\section{CONCLUSIONS}

The study identify the usefulness of both the methods in building extraction as building footprints are the basic layer or component for any smart planning and designing activities. The study has presented comparison of two segmentation methods for extraction of building features, Rule based feature extraction of ENVI and Multiresolution Segmentation algorithm of eCognition. The rule sets were derived for the efficient extraction of buildings for both the methods using hit $\&$ trial method and literature studies. For accuracy assessment, confusion matrix was generated. For performance comparison approach proposed by Jamet, (1997) is used.The result of extracted building from Multiresolution Segmentation algorithm shows overall more accuracy compared to Rule based feature extraction algorithm, as the False Negative Rate of ENVI is very high. The buildings were extracted with overall accuracy of 88.9\%, and Kappa Index of 0.813. Urban features such as buildings and roads extracted by these geospatial techniques can be used for various applications such as base map preparation of a city for physical or social infrastructure planning, smart city planning, development and management.

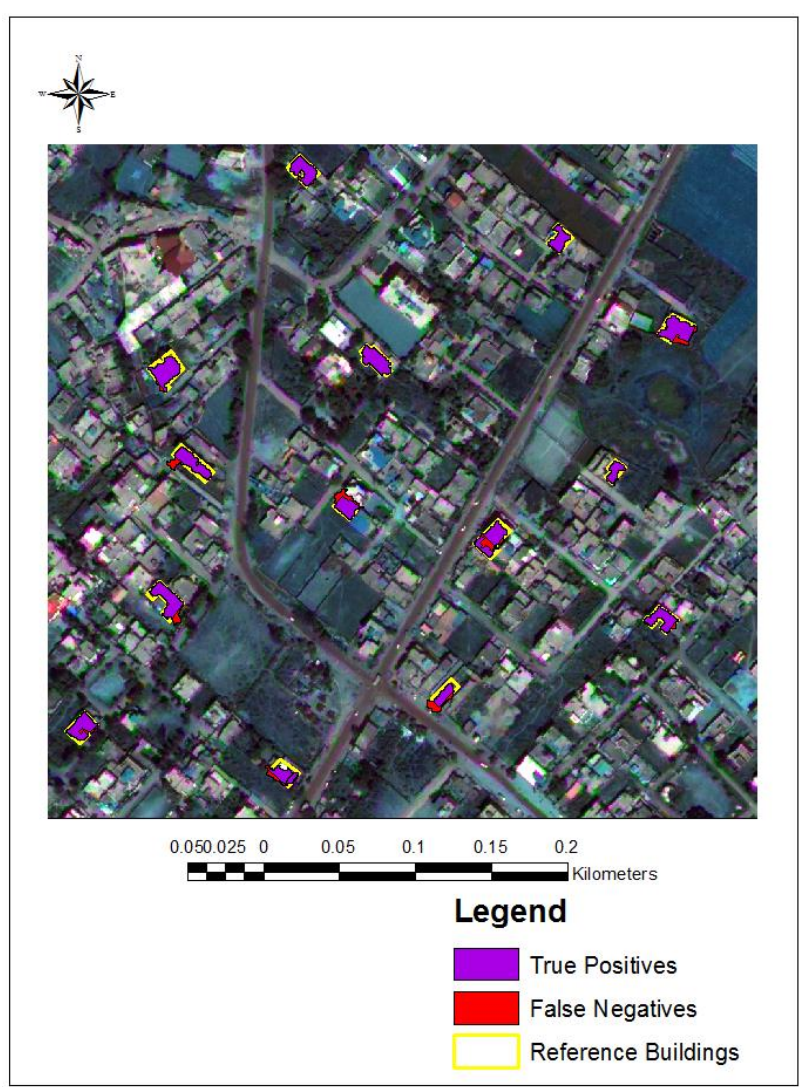

Figure 13 Accuracy assessment for Multi-Resolution algorithm

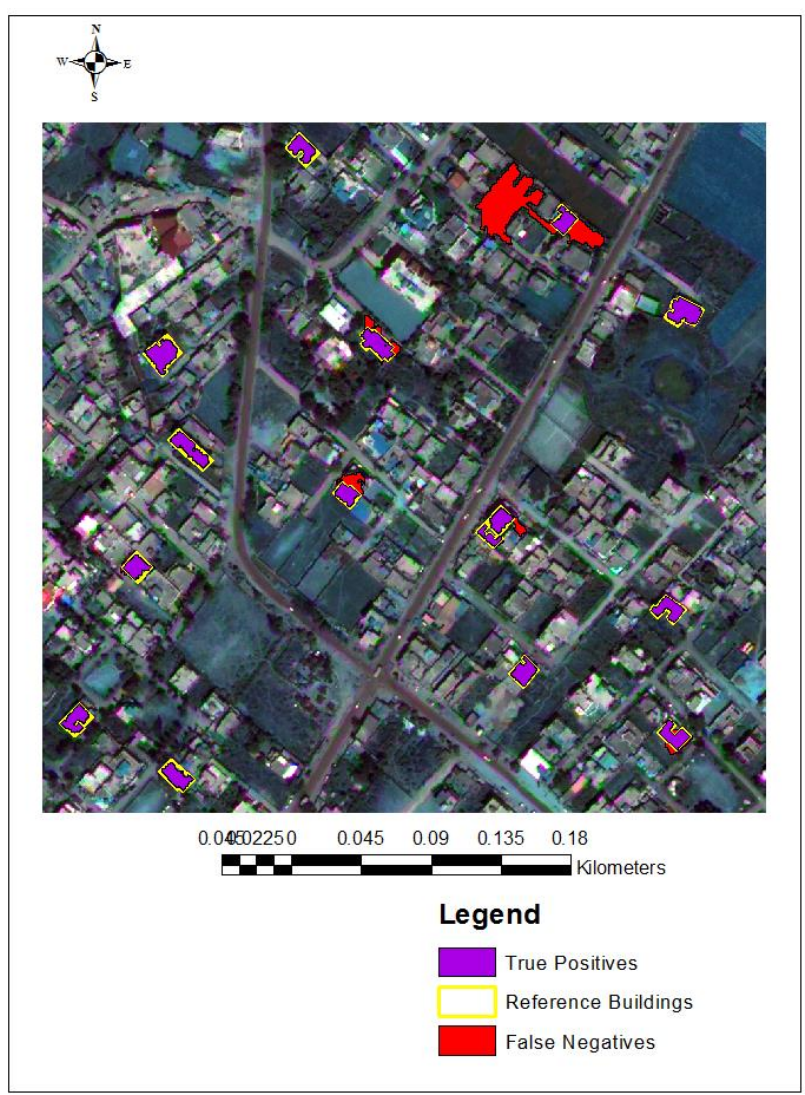

Figure 14 Accuracy assessment for Rule based feature extraction algorithm 


\section{REFERENCES}

Belgiu, M. and Drăguţ, L. (2014) 'Comparing supervised and unsupervised multiresolution segmentation approaches for extracting buildings from very high resolution imagery', Isprs Journal of Photogrammetry and Remote Sensing. Elsevier, 96, pp. 67-75. doi: 10.1016/j.isprsjprs.2014.07.002.

Benediktsson, J. A., Pesaresi, M. and Arnason, K. (2003) 'Classification and Feature Extraction for Remote Sensing Images From Urban Areas Based on Morphological Transformations', IEEE Transactions on Geoscience and Remote Sensing, 41(9), pp. 1940-1949. doi: 10.1109/TGRS.2003.814625.

Census of India, 2. (2015). Census 2011. Retrieved from Dehradun City Census 2011 data: https://www.census2011.co.in/census/city/23-dehradun.html

Itt (2008) 'ENVI feature extraction module user's guide', Computing, p. 78.

Jamet, C. H. (1997). Evaluation of Automatic Road Extraction. International Archives of Photogrammetry and Remote Sensing, 47-56.

Jiang, N. et al. (2008) 'Semi-automatic building extraction from high resolution imagery based on segmentation', in 2008 International Workshop on Earth Observation and Remote Sensing Applications, pp. 1-5. doi: 10.1109/EORSA.2008.4620311.

Jumlesha, S. K. et al. (2012) 'Automatic Urban Feature Extraction Using', International Journal of Engineering Research and Applications (IJERA), 2(3), pp. 221-225.

Singh, D. et al. (2012) 'Building extraction from very high resolution multispectral images using NDVI based segmentation and morphological operators', 2012 Students Conference on Engineering and Systems, pp. 1-5. doi: 10.1109/SCES.2012.6199034

Uttarakhand, G. o. (2018). District Dehradun. Retrieved from District Administration Dehradun: https://dehradun.gov.in/history/ 